\title{
BMJ Open Understanding quality of contraceptive services from women's perspectives in Gujarat, India: a focus group study
}

\author{
Kelsey Holt (D) , ${ }^{1}$ Bella Vasant Uttekar, ${ }^{2}$ Reiley Reed, ${ }^{1,3}$ Madeline Adams, ${ }^{4}$ \\ Lakhwani Kanchan, ${ }^{2}$ Ana Langer, ${ }^{4}$ Sandhya Barge ${ }^{2}$
}

To cite: Holt K, Uttekar BV, Reed $\mathrm{R}$, et al. Understanding quality of contraceptive services from women's perspectives in Gujarat, India: a focus group study. BMJ Open 2021;0:e49260. doi:10.1136/ bmjopen-2021-049260

- Prepublication history for this paper is available online. To view these files, please visit the journal online (http://dx.doi org/10.1136/bmjopen-2021049260).

Received 20 January 2021 Accepted 21 September 2021

Check for updates

(c) Author(s) (or their employer(s)) 2021. Re-use permitted under CC BY-NC. No commercial re-use. See rights and permissions. Published by BMJ.

${ }^{1}$ Department of Family \& Community Medicine, University of California, San Francisco, California, USA

${ }^{2}$ Centre for Operations Research and Training, Vadodara, Gujarat, India

${ }^{3}$ School of Social Welfare, University of California, Berkeley, California, USA

${ }^{4}$ Global Health and Population, Harvard University T H Chan School of Public Health, Boston, Massachusetts, USA

Correspondence to

Dr Kelsey Holt;

kelsey.holt@ucsf.edu

\section{ABSTRACT}

Objectives Understanding quality of contraceptive care from clients' perspectives is critical to ensuring acceptable and non-harmful services, yet little qualitative research has been dedicated to this topic. India's history of using incentives to promote contraceptive use, combined with reports of unsafe conditions in sterilisation camps, make a focus on quality important. The study objective was to understand women's experiences with and preferences for contraceptive counselling and care in the public sector in India.

Design Qualitative study using eight focus group discussions (FGDs). FGDs were thematically analysed using a framework approach.

Setting Rural and urban areas in one district in Gujarat. Participants 31 sterilisation and 42 reversible contraceptive users who were married and represented different backgrounds. Inclusion criteria were: (1) female, (2) at least 18 years and (3) receipt of contraception services in the last 6 months from public health services.

Results Providers motivate married women to use contraception and guide women to specific methods based on how many children they have. Participants found this common practice acceptable. Participants also discussed the lack of counselling about reversible and permanent options and expressed a need for more information on side effects of reversible methods. There were mixed opinions about whether compensation received for accepting longterm methods affects contraceptive decision making. While many women were satisfied with their experiences, we identified minor themes related to provider coercion towards provider-controlled methods and disrespectful and abusive treatment during sterilisation care, both of which require concerted efforts to address systemic factors enabling such experiences.

Conclusions Findings illuminate opportunities for quality improvement as we identified several gaps between how women experience contraceptive care and their preferences, and with ideals of quality and rights frameworks. Findings informed adaptation of the Quality of Contraceptive Counselling Scale for India, and have implications for centring quality and rights in global efforts.

\section{INTRODUCTION}

High-quality contraceptive counselling and method provision are fundamental to a human rights based approach to family

\section{Strengths and limitations of this study}

This study investigated rural and urban women's experiences with and preferences for contraceptive counselling and care in Gujarat, India.

- This is one of few studies from a low-income or middle-income country that uses qualitative methods to understand the client perspective on quality of care.

- This is one of few studies that investigates women's experiences with sterilisation care in India, a country with a long history of high rates of use of this method.

- This study is limited by its geographical focus on only one state in India, a large country with much regional diversity.

- Unmarried women who do not frequently seek contraceptive services in the public system were not included in this study.

planning care. ${ }^{1}$ Understanding quality in-depth from the user perspective is critical to ensuring services are acceptable and not harmful and is essential to tailoring quality monitoring and improvement strategies to women's preferences for care in a given setting.

In India, a history of using incentives for both women and providers to promote contraceptive use and population control, ${ }^{2-4}$ combined with decades of reports of unsafe conditions especially in sterilisation camps, ${ }^{34}$ makes a focus on quality in family planning care particularly critical. Around $47.8 \%$ of married couples are current users of some method of modern contraception. ${ }^{5}$ Though the method mix is shifting, with increased use of reversible methods in recent years, ${ }^{5}$ over two-thirds of contraceptive users in India still adopt permanent, female sterilisation. ${ }^{6}$ The median age of women who undergo sterilisation in India is 25.7, and women with lower levels of education and greater fecundity are more likely to be sterilised. ${ }^{5}$ Though the country has moved away 
from a target-based approach to family planning towards a community-based reproductive health service provision approach, women and healthcare providers are still paid financial compensation to uptake and provide postpartum intrauterine devices (IUDs) and sterilisation and experts have called for a greater shift towards a focus on quality of care. ${ }^{7}$

For both permanent and reversible contraception, high-quality counselling entails providing individuals with personalised information about available methods and unbiased decision support to select the method that will best suit their needs. ${ }^{8-10}$ For provider-dependent methods that require procedures-including IUDs and sterilisation-another critical component of service quality is respectful treatment and sanitary conditions during the procedure.

Research with women about their experiences with counselling and procedures can help to identify gaps in quality of care and monitor for potential human rights abuses. The family planning field has a long history of measuring users' perspectives on quality, primarily using an exit survey methodology. ${ }^{11}$ Fewer studies have explored women's perspectives on quality in-depth through use of qualitative methods. Limited qualitative data from India have documented women's experiences of pressure to accept contraception and lack of counselling on method options, side effects and what to expect with sterilisation procedures. ${ }^{1213}$

Furthermore, asking women not only about their experiences with care, but also their preferences, is important to guide efforts to make contraceptive care better meet individuals' needs and expectations as they themselves define them. Scarce data from North America suggest many women value a shared decision-making approach to counselling, wherein providers solicit individual preferences and needs and make suggestions for methods that may work best for them. ${ }^{14}{ }^{15}$ Limited research also suggests that, conversely, some women prefer that providers select the best method based on tests or professional opinion, ${ }^{14} 16$ which runs contrary to the nature of contraceptive decision making as preference sensitive. ${ }^{17}$ Overall, however, very little is known about women's preferences for contraceptive care in low-ncome and middleincome country settings. Understanding how women in India view their role in contraceptive decision making is critical to tailoring efforts to provide high quality, rightsbased information and care.

In this study, our objective was to investigate women's experiences with and preferences for contraceptive counselling and care in the public sector in rural and urban areas of Gujarat, India, using a qualitative approach. This research was conducted as part of a larger project to develop the Quality of Contraceptive Counselling (QCC) Scale for quantitative measurement of women's perceptions of the QCC in India, Ethiopia and Mexico. ${ }^{18}$ These findings informed adaptation of the QCC Scale for India (results forthcoming), and also have implications for regional efforts to improve the QCC in India as well as adding to the international evidence base on preferences for contraceptive care among women worldwide.

\section{METHODS}

\section{Study design and population}

We conducted eight focus group discussions (FGDs) with female contraceptive users in one District of Gujarat State in India between August and October 2017. FGDs were appropriate for this study given that our goal was to understand norms around how contraception services are perceived and preferences for how they could be improved among women served by the public sector.

Inclusion criteria were: (1) female, (2) at least 18 years old and (3) receipt of some form of contraception services in the last 6 months from public health services. Half of the FGDs took place with women recruited in rural areas and half took place with women in urban areas. To increase homogeneity among focus groups, women were separated into two categories: those who had initiated a reversible method of contraception within the last 6 months; and those who had been sterilised. In order to have at least two groups with each category in each geographic area, we determined a priori that an appropriate sample size would be eight FGDs.

A research team based at Centre for Operations Research and Training (CORT) collected all data.

\section{Recruitment and study procedures}

To recruit study participants, Accredited Social Health Activists (ASHAs) distributed fliers describing the FGDs to women in their catchment areas who had received contraceptive services in the last 6 months across eight selected facilities (four rural, four urban). To avoid bias in who was invited to participate, ASHAs were instructed to invite all women in their area whom they were aware were using contraception. When women expressed interest in the study, study staff screened them and enrolled eligible participants. All participants provided informed consent before taking part in an FGD.

Each FGD included 7-13women and lasted around an hour and took place in the local health centres' private conference rooms. Participants' travel expenses were reimbursed at approximately US $\$ 2.50$ and refreshments were provided during the FGDs. The sessions included a note-taker and moderator, who followed a semistructured FGD guide centred on women's experiences with and preferences for contraceptive counselling and procedures. Both moderators (BVU and SB) are female and experienced social science researchers from India with decades of experience conducting qualitative research. When introducing themselves to participants, they made clear they were from a research organisation and not representing the public health system.

FGD topics included: (1) experiences talking to healthcare providers about contraceptive options in the public sector, including probes around whether experiences were good or not good and why, and around what specific 
information is generally given; (2) perspectives on what defines a 'good' versus a 'not so good' interaction with providers about contraception; (3) what an ideal interaction should look like, including probes about what specifically providers should ask women before providing a method and what information should be given; (4) preferences for healthcare provider involvement in decision making, including a probe about whether the client or provider should be the ultimate decision-maker; (5) whether monetary compensation plays a role in method decision making and (6) perspectives on elements of the QCC Framework ${ }^{9}$ (privacy, confidentiality, respect, trust, empathy and non-discrimination) which guided development of the QCC Scale, through a series of probes about what these mean to them and their experiences with providers ensuring or not ensuring each one.

A brief survey was administered to participants to collect information on socio-demographic characteristics, current contraceptive method used and receipt of information about other methods during their provider interactions.

The FGDs were conducted in Gujarati and audio recorded, then transcribed in Gujarati and translated into English by members of the CORT research team.

\section{Analysis}

We employed a framework approach to qualitative analysis, beginning with a list of deductive codes derived from the QCG Framework and the FGD guide and developing additional inductive codes as thematic analysis progressed. ${ }^{19} \mathrm{MA}$ and RR developed the initial codebook with input from KH. After application of the codebook by RR to several transcripts, KH reviewed the coding and discussed discrepant opinions with RR in iterative rounds until agreement was reached and the codebook was finalised. RR completed all coding using NVivo software. Code summaries were created by RR, and synthesised by $\mathrm{KH}$, with input from BVU, KL and SB, to provide descriptions of major and minor themes identified related to women's experiences and expressed preferences for contraceptive counselling and procedures. When reviewing code summaries, we looked for any relevant differences in themes between FGDs conducted with women in rural versus urban areas given the different health system and cultural contexts between these settings. We did not examine potential differences by individual characteristic, such as caste or educational background, because we were focused on group-level norms. Our analysis revealed evidence of thematic saturation as many of our themes related to experiences with and preferences for counselling were identified in numerous FGD transcripts.

Survey findings were analysed using basic descriptive statistics.

\section{Patient and public involvement statement}

Patients or the public were not involved in the design, or conduct, or reporting or dissemination plans of our research.

\section{RESULTS}

\section{Participant characteristics}

Seventy-three women (31 using sterilisation and 42 using reversible contraception) participated in the eight focus groups. Mean age was 28 years, and women from a variety of educational backgrounds and castes participated (table 1). All participants were married, most dedicated themselves to household chores $(64 \%)$, and the average number of children was 2 .

\section{Survey results: contraceptive use and receipt of information during counselling}

Reversible contraceptive users used either condoms, pills or the IUD (table 2). Survey findings revealed that $19 \%$ of sterilisation users and $52 \%$ of reversible method users reported being informed about other methods when they received their current method, and $3 \%$ of sterilisation users and $21 \%$ of reversible method users received information on emergency contraception.

\section{Focus group results: experiences with and preferences for contraceptive counselling and procedures}

We organise our findings from analysis of FGDs into themes grouped by the following topics: contraceptive counselling, financial compensation, privacy and confidentiality during counselling and procedures, interpersonal relationships and trust in providers, and experiences with IUD insertion and tubal ligation procedures.

\section{Contraceptive counselling}

There was a common understanding among participants that it was common, acceptable and expected that ASHAs and other providers would motivate married women to use contraception and tailor information based on how many children a woman already had; specifically, sterilisation for women with two children (or, as some pointed out, three if the couple had two girls and wanted a boy), the copper IUD for spacing children, and pills and condoms for prior to having children or for spacing. The following quote summarises this phenomenon: 'If we have two children then they make us understand about the operation or if [we] have one child then they make us understand about copper T, condom, or any other method.' (Reversible method, urban) The phrase 'make us understand' was commonly used to describe positive interactions with providers and reflected women's acceptance of such directive counselling. Not surprisingly, given the approach of guiding women towards particular methods, no one described being informed about both sterilisation and reversible methods or receiving counselling about choosing among all methods. As corroborated by survey results in table 2 , sterilisation and reversible method users commonly reported not receiving information about other options. We did not find any notable differences in findings related to counselling experiences between rural and urban groups.

When asked what defines a 'good' versus a 'not so good' interaction with a provider about contraception and what 
Table 1 Profile of the participants in the FGDs

\begin{tabular}{|c|c|c|c|c|c|c|}
\hline & Steri & & Reve & & Tots & \\
\hline & $\mathbf{N}$ & $\%$ & $\mathbf{N}$ & $\%$ & $\mathbf{N}$ & $\%$ \\
\hline Total no of participants & 31 & 100.0 & 42 & 100.0 & 73 & 100.0 \\
\hline Rural & 15 & 48.4 & 21 & 50.0 & 36 & 49.3 \\
\hline Urban & 16 & 51.6 & 21 & 50.0 & 37 & 50.7 \\
\hline Age of the focus group participa & & & & & & \\
\hline $20-24$ & 10 & 32.3 & 11 & 26.2 & 21 & 28.8 \\
\hline $25-29$ & 8 & 25.8 & 16 & 38.1 & 24 & 32.9 \\
\hline $30-34$ & 8 & 25.8 & 9 & 21.4 & 17 & 23.3 \\
\hline $35-39$ & 3 & 9.7 & 4 & 9.5 & 7 & 9.6 \\
\hline $40-44$ & 1 & 3.2 & 2 & 4.8 & 3 & 4.1 \\
\hline Not specified & 1 & 3.2 & 0 & 0.0 & 1 & 1.4 \\
\hline Mean age & 27.8 & & 28.3 & & 28.1 & \\
\hline Education of the participants & & & & & & \\
\hline Illiterate & 4 & 12.9 & 1 & 2.4 & 5 & 6.8 \\
\hline Primary (1-5) & 4 & 12.9 & 3 & 7.1 & 7 & 9.6 \\
\hline Middle (6-8) & 12 & 38.7 & 9 & 21.4 & 21 & 28.8 \\
\hline Secondary $(9-10)$ & 9 & 29.0 & 15 & 35.7 & 24 & 32.9 \\
\hline Higher Secondary (11-12) & 1 & 3.2 & 3 & 7.1 & 4 & 5.5 \\
\hline Graduation (1-15) & 1 & 3.2 & 11 & 26.2 & 12 & 16.4 \\
\hline Caste of the participants & & & & & & \\
\hline Schedule caste $(\mathrm{SC})^{\star}$ & 4 & 12.9 & 14 & 33.3 & 18 & 24.7 \\
\hline Schedule tribe (ST) & 12 & 38.7 & 6 & 14.3 & 18 & 24.7 \\
\hline Other backward class (OBC) & 2 & 6.5 & 10 & 23.8 & 12 & 16.4 \\
\hline General & 13 & 41.9 & 12 & 28.6 & 25 & 34.2 \\
\hline Work status & & & & & & \\
\hline Worked outside the home & 10 & 32.3 & 10 & 23.8 & 20 & 27.4 \\
\hline Work for household business & 4 & 12.9 & 2 & 4.8 & 6 & 8.2 \\
\hline Household chores & 17 & 54.8 & 30 & 71.4 & 47 & 64.4 \\
\hline Marital status & & & & & & \\
\hline Currently married & 31 & 100.0 & 42 & 100.0 & 73 & 100.0 \\
\hline Mean total no of children & 2.4 & & 1.6 & & $1 . \varsigma$ & \\
\hline
\end{tabular}

*The SC and ST are designated by the government as the most disadvantaged groups in India; OBCs are also educationally or socially disadvantaged though less so than the SC and ST groups. Those in the general caste are relatively more advantaged.

FGDs, focus group discussions.

an ideal interaction would look like, women particularly in the rural groups often struggled to articulate their preferences. After much probing, a common theme emerged in both reversible and permanent contraception groups around provision of accurate, detailed information about the one method a person had come for or that the provider was recommending. A minor theme was participants wanting information about multiple methods, as some women in urban settings did express the importance of learning about the advantages and disadvantages of multiple methods before selecting a method. In terms of what type of information women wanted, for reversible methods, women reported wanting proactive information on side effects and how to use the method (eg, how long to wait before having sex, when to pause using pills for menstruation) or, less commonly, whether the method would prevent against HIV infection. Occasionally, women would mention that in addition to explaining methods to women, providers should also understand women's health history or life situation in order to tailor counselling, though this was a minor theme. In one of the urban groups among reversible contraception users, respondents discussed the importance of telling clients that they can discontinue pills or have their IUD removed if they do not like it (in this group, many participants commented that, on the contrary, they were told not to 
Table 2 Participants' contraceptive use and receipt of information during counselling

\begin{tabular}{|c|c|c|c|c|c|c|}
\hline & \multicolumn{2}{|c|}{ Sterilisation } & \multicolumn{2}{|c|}{ Reversible } & \multicolumn{2}{|c|}{ Total } \\
\hline & $\mathbf{N}$ & $\%$ & $\mathbf{N}$ & $\%$ & $\mathbf{N}$ & $\%$ \\
\hline Total no of participants & 31 & 100.0 & 42 & 100.0 & 73 & 100.0 \\
\hline Female sterilisation & 31 & 100.0 & 0 & 0.0 & 31 & 42.5 \\
\hline IUD & 0 & 0.0 & 9 & 21.4 & 9 & 12.3 \\
\hline Condom & 0 & 0.0 & 17 & 40.5 & 17 & 23.3 \\
\hline Informed about other methods & 6 & 19.4 & 22 & 52.4 & 28 & 38.4 \\
\hline Told about emergency contraceptive pills & 1 & 3.2 & 9 & 21.4 & 10 & 13.7 \\
\hline
\end{tabular}

IUD, intrauterine device.

remove their IUD too soon after receiving it because their body may adjust to the IUD after time).

In sterilisation groups, participants specifically discussed the lack of information about sterilisation advantages and disadvantages provided to clients in advance of the procedure. ASHAs were described as providing the most information to women who had decided to undergo sterilisation, particularly in the form of emotional support letting them know that the procedure would be fast and that they should not be scared. In one group, there was a discussion of the fact that, while ASHAs provide such support preprocedure, there is not sufficient support at the time of the procedure. As one participant in this group noted: 'ASHA worker only make us understand everything, after going to hospital, nothing is told.' (Permanent method, urban)

Some participants in all the sterilisation groups shared that they were influenced by the provider suggesting sterilisation as a method, either because the provider viewed reversible methods as having undesirable side effects, because reversible methods could fail and lead to the need for abortion, or, explicitly because they viewed the woman as having enough children; for example: 'I had undergone cesarean for two times, so doctor asked me to go for operation (sterilization)' (Permanent method, urban). As described above, women mostly described this influence in a neutral way, without expressing displeasure for the directive counselling. A minor theme was expressing a sense of feeling pressure towards sterilisation; for example, one person described being encouraged by the ASHA to schedule an operation, and implied that she was being pressured into it:

ASHA asked me 2-3 times that do you want to come for the operation, I say yes that I am coming; and I fixed the date also but at the end time I got scared and then canceled my appointment. (Permanent method, urban)

Respondents in both rural and urban areas overwhelmingly indicated that while providers can share contraceptive information and make suggestions, they should not make the final decision about women's contraceptive choices. For example, one respondent stated, 'Should take [the decision] by her own self, Madam [ASHA or service provider] only explains to us but the decision should be ours only' (Reversible method, rural).

\section{Financial compensation}

When asked how the common practice of using payments for women who adopt contraception influences decision making, participants expressed mixed opinions. Many participants in most groups felt strongly that when women receive money on uptake of an IUD or being sterilised, this has no impact on their decision to begin using the method (as one person from an urban reversible contraceptive method group stated: 'There is no importance of incentives in the decision of family planning method'). A minor theme was that compensation does influence women's decisions when they are poor, and in one group, participants overwhelmingly felt that government should not provide compensation to women for contraceptive use, as demonstrated by this quote: 'Yes government pays us for using Copper-T or sterilization ... And we don't think that government should give this money as it is our responsibility of having or not having children.' (Reversible method, urban)

\section{Privacy and confidentiality}

When asked about the importance of privacy and confidentiality, respondents commonly described privacy as essential to being able to have open conversations with their provider. For example, one said: 'If I am sitting alone with you I can share with you my problems or what is going in my mind but if there are two or three people around us I will not be able to share anything with you. We cannot talk in front of everyone.' (Reversible method, urban) Participants discussed the importance of providers keeping services confidential from a client's husband or mother-in-law if the client prefers it that way, as described in this quote:

Sometimes it might be possible that women want to take pills but the mother-in-law does not allow her to 
take them, so we tell the service provider, 'you give pills to me but you should not tell to anyone or anyone should not come to know about it'. (Reversible method, rural).

Respondents in multiple groups reported that ASHAs do usually attempt privacy during home visits by conducting the visit outside or in a separate room, particularly when male family members are present. However, one group discussed their impression that confidentiality is not important to providers, and another discussed their experiences with providers giving information about family planning in front of others.

Several participants also raised privacy concerns related to having received contraceptive procedures in a hospital without privacy from men or having received preprocedure injections in line with other women.

\section{Interpersonal relationships and trust in providers}

Participants were asked to describe ideal elements of relationships with providers that demonstrate respect and build trust. In addition to desiring that providers speak to them 'nicely,' 'politely' or 'gently', groups commonly discussed valuing comprehensive information and advice. Ensuring privacy and confidentiality was also linked to development of trusting relationships. For example, one participant said, 'When we talk to her [provider] and she does not tell anyone, then our trust is developed.' (Reversible method, urban)

The importance of providers trying to understand clients' unique situations by asking questions and demonstrating respect for individual needs was another theme. For example, one person said, 'To give respect is to show that the person has some value' (Reversible method, urban). Another stated that she valued when a provider, 'Does not get angry, and explains to us in our way so we can understand'. (Permanent method, urban). Another said, 'Yes they respect us when we meet and ask us how are we, how is our health? They frequently ask us these questions'. (Reversible method, urban)

The importance of all of these elements was highlighted by participants who described in several groups the fact that they do not seek contraceptive care when providers fail to demonstrate respect or engender trust. As one participant stated: 'We will not come a second time [if providers do not demonstrate respect].' (Reversible method, rural).

When asked, respondents largely indicated that there is no longer discrimination based on caste, socioeconomic status or education-level, as there had historically been, though in some groups, there was mention of ongoing discrimination occurring in small villages.

\section{Experiences with IUD insertion and tubal ligation procedures}

Several groups discussed the important role of ASHAs in supporting women undergoing tubal ligations, and that they often provide support to women before and after the procedure, which many women expressed appreciation for.
During the tubal ligation procedure, some women in sterilisation groups described good experiences; for example, one said, 'Service provider talked with me nicely during operation, after operation also helped nicely to get out of the room and made us sit for a while. That all we liked and is a good treatment.' (Permanent method, rural)

However, women in the two urban sterilisation groups also discussed several aspects of their experiences that reflected disrespectful or abusive care. Related to the complaints about lack of privacy (described above), some described crowding as a problem. For example, one said: 'Once when I went to medical college (tertiary hospital) there was no space on the bed and 2-3 people together were made to lay down on the same bed, there is not enough space.' (Permanent method, urban) Participants also discussed experiences of providers being disrespectful or getting angry if women express pain during the procedure, as described by this participant: 'They get angry throughout the operation; even if someone shouts then he gets angry and says, 'Shut up.' (Permanent method, urban)

Other participants described lack of postoperative support, including not having beds to recover in and feeling rushed during recovery. As one participant described:

As soon as the operation is done they make us get down from the stretcher. We feel a little bit hurting, but in this period we have to get down immediately after the operation...they don't provide a bed, directly we have to get down on the floor and rest there. (Permanent method, urban)

When asked their thoughts about compensation for sterilisation, a participant in this group responded that it was in fact the lack of follow-up care that was troubling:

It's not about money but it's that we don't know what is happening, and what a problem is, and whether everything is alright or not...so they should call after operation for follow-up and checkup. (Permanent method, urban)

Related to IUD insertions, two groups in urban areas brought up various forms of coercion or force to use IUDs that happened to them or people they know. One group discussed this at length, with multiple participants sharing experiences of having an IUD inserted after delivery without their knowledge or being told by providers that they would be scolded for removing an IUD too early. Respondents in this group agreed that, "After delivery, they compulsorily insert Copper T [IUD].' (Reversible method, urban) One person shared her experience as follows: 'Without asking me they inserted copper T. Forcefully they insert copper $\mathrm{T}$ at higher level public hospitals. I said no only for inserting copper T, but they told it is good if you will insert it.' (Reversible method, urban) She reported still having it at the time of the focus group. Describing the pressure to not remove an IUD early, one 
participant described: 'they do say like this; that copper T is for three years if you will come before that for removing it then we will scold you.' (Reversible method, urban)

\section{DISCUSSION}

Findings from this set of focus groups with women in Gujarat, India, illuminate a number of gaps between how women experience contraceptive counselling and contraceptive procedures (including IUD insertions and tubal ligations) in the public sector and their preferences for such care, as well as with the ideals laid out in quality of care and rights frameworks related to informed choice and autonomy. ${ }^{17}$ These results highlight the need for continued investment in policy and programmatic efforts to ensure provision of high quality, rights-based contraceptive services to women in India.

Related to counselling, in line with limited other qualitative research from India, ${ }^{12}$ participants commonly discussed not receiving full information about method options, and indeed only $19 \%$ of sterilisation users and $52 \%$ of reversible method users reported being informed about other methods when they received their current method. Particularly for women undergoing sterilisation, because of the permanent nature of this method, this is an important area for improvement to ensure that women have the opportunity to make informed choices between available permanent and reversible methods, even in a country like India where the method mix is more limited than in other places. Moving towards more comprehensive, neutral counselling about reversible and permanent method options may take concerted policy and programmatic efforts given the long history of reliance on sterilisation in India and the tendency to accept a directive approach to counselling that was reflected among our focus group findings with women. Further, women in our study-particularly in rural areas-often struggled to articulate their preferences for additional information and support from providers, and accepted a more directive approach to counselling; this highlights the importance of systemic mechanisms for holding services accountable for providing high-quality counselling to support informed choice when women may not be empowered to demand such care.

Desiring more information about chosen reversible methods-particularly related to side effects-was another common theme that emerged, similar to other research on women's preferences for contraceptive counselling which has found that women commonly want more information on potential side effects and side effect management than they receive. ${ }^{14}{ }^{15} \mathrm{~A}$ minor theme was women expressing a desire for more personalised counselling based on an understanding of women's priorities and life situations, which is in line with guidelines for high-quality contraceptive counselling. ${ }^{9} 17$

Participants expressed a desire for privacy and confidentiality when discussing contraception with providers, particularly in the context of home visits from ASHAs (though for the most part, women felt that ASHAs already do a good job at making sure women can speak freely by holding conversations outside when other family members are present), in line with human rights and quality frameworks. ${ }^{1} 810$ Women in our study also highlighted the importance of interpersonal relationships with providers in engendering their trust. Consistent with this finding, a new Lancet framework for high-quality health systems includes trust in the system as a key output of high-quality care. ${ }^{20}$ Particularly in the context of contraceptive care, making sure that women feel respected and listened to is critical to ensuring they feel comfortable engaging with the system when needed for reproductive healthcare.

Our finding of a minor theme related to women experiencing pressure or force to use IUDs or sterilisation from providers is concerning, particularly the stories participants shared about non-consensual IUD insertions. Further investigation into the role of systems level factors, such as the continuing use of financial compensation for providers for postpartum IUDs and sterilisation, ${ }^{7}$ and sociocultural norms related to childbearing in encouraging providers to pressure or force women into contraceptive use is needed to gain a better understanding of why this phenomenon is occurring in the present context and to identify strategies for promoting informed choice and autonomy in contraceptive care. While only a minority of women in our study felt that compensation for contraceptive use influences decision making, payment for uptake of contraceptive methods is clearly in conflict with human rights principles and should be discontinued. ${ }^{21}$ Other recent studies from FP2020 countries have found evidence of provider pressure to use certain methods ${ }^{22}$; the new iteration of the international FP2020 movement offers an opportunity to refocus on informed choice and autonomy in contraceptive care. ${ }^{23}$

Finally, this study is unique in exploring women's experiences and preferences for sterilisation procedures. Our findings reveal that, while ASHAs played a critical role in preparing women for the procedure and in some cases caring for them afterwards, women receive limited information and support during the actual procedure and often experience lack of privacy and crowding, in line with prior research conducted in Bihar. ${ }^{13}$ The experiences of women with scolding during the procedure, and lack of bed space for recovery, are reminiscent of findings from research on women's experiences during labour and delivery in many settings, including India. ${ }^{24}$ It is unclear whether the fact that such negative experiences were mainly reported among urban groups reflects a difference in the quality of services provided or expectations of women in those areas. Regardless, coordinated efforts across the continuum of reproductive, maternal, newborn, child, and adolescent health are needed to address structural and interpersonal barriers to respectful care and treatment. ${ }^{25}$

This study is limited by its geographical focus on only one state in India, a large country with much regional diversity. We also acknowledge that, while the vast majority 
of permanent method users receive services in the public sector in Gujarat, less than half of reversible contraceptive users do, and thus, we are limited in our ability to comment on women's experiences with reversible method counselling and provision in the commercial and private sectors where the majority of women access services. Finally, we are limited in our recruitment approach. The most feasible approach to contacting women who had recently received contraceptive counselling was to rely on ASHAs. In the current approach to community-based reproductive health service provision in Gujarat, ASHAs are supposed to regularly visit all households in their catchment area, and in our study they were asked to reach out to all women who they knew had started contraception within the last 6 months. However, reliance on ASHAs could nonetheless have resulted in under-representation of women who may have had negative relationships with the ASHA in their area and a skew in our results towards inclusion of more positive experiences with contraception services. This recruitment approach also resulted in under-representation of unmarried women who do not frequently seek contraceptive services in the public system due to cultural norms against use of contraception before marriage and who also may have more negative experiences due to potential experience of stigmatisation or discrimination when seeking care. Despite these limitations, our findings are valuable given the dearth of available qualitative data about women's experiences and preferences for contraceptive care.

\section{CONCLUSION}

In conclusion, this study offers the opportunity to hear from women in India themselves what matters to them related to contraceptive counselling and procedures, and where there is room for improvement in public sector service delivery. Similar to other qualitative studies on women's experiences with contraceptive care, our findings shed light on the need for improvements related to fulfilment of rights principles of informed choice-namely a need for unbiased counselling on method options and more information provision related to side effects-and autonomy, given to our finding that some women experienced coercion towards provider-controlled methods. We additionally found the need for better ensuring respectful treatment during sterilisation procedures. The new iteration of the FP2020 movement offers an opportunity for the global community, including in India, to refocus on quality and rights rather than numeric targets related to contraceptive uptake. Mixed methods research with clients in different settings-including qualitative research like this study and quantitative research using human rights-based quantitative instruments such as the QCC Scale to more widely gauge quality of client experience-will continue to be essential to such efforts.

Acknowledgements In addition to thanking the participants in this study for sharing their time and perspectives with us, we would like to thank Jashoda
Sharma for her assistance with data collection and Shashi Sarnaik for her assistance with editing the final version of this manuscript.

Contributors $\mathrm{KH}$ and $\mathrm{AL}$ conceived of the study; BVU, SB, KL and KH designed study instruments; BVU, SB and KL conducted data collection; KH, MA, RR, SB, BVU and $\mathrm{KL}$ conducted the analysis; $\mathrm{KH}$ and $\mathrm{RR}$ drafted the manuscript; $\mathrm{AL}, \mathrm{SB}, \mathrm{BVU}, \mathrm{KL}$ and MA provided substantive feedback on the manuscript.

Funding This work was supported by the David and Lucile Packard Foundation (Grant number 2016-64353).

Competing interests None declared.

Patient and public involvement Patients and/or the public were not involved in the design, or conduct, or reporting, or dissemination plans of this research.

Patient consent for publication Not required.

Ethics approval The study was approved by the Harvard T.H. Chan School of Public Health institutional review board (IRB) (Harvard ID IRB17-0861) and CORT's IRB (ID N/A). All participants provided informed consent before taking place in the study.

Provenance and peer review Not commissioned; externally peer reviewed.

Data availability statement The data that support the findings of this study are available from the corresponding author on reasonable request.

Open access This is an open access article distributed in accordance with the Creative Commons Attribution Non Commercial (CC BY-NC 4.0) license, which permits others to distribute, remix, adapt, build upon this work non-commercially, and license their derivative works on different terms, provided the original work is properly cited, appropriate credit is given, any changes made indicated, and the use is non-commercial. See: http://creativecommons.org/licenses/by-nc/4.0/.

ORCID iD

Kelsey Holt http://orcid.org/0000-0001-9093-4322

\section{REFERENCES}

1 WHO. Ensuring Human Rights in the Provision of Contraceptive Information and Services: Guidance and Recommendations. Geneva: : World Health Organization, 2014. Available: http://www.ncbi.nlm. nih.gov/books/NBK195054/ [Accessed 30 Sep 2020].

2 Visaria L, Ved RR. India's Family Planning Programme: Policies, practices and challenges. Routledge, 2016.

3 HRW India - Human Rights Watch. India: target-driven sterilization harming women. hum. rights watch, 2012. Available: https://www. hrw.org/news/2012/07/12/india-target-driven-sterilization-harmingwomen [Accessed 30 Sep 2020].

4 Mohanty S, Bhalla N. Indian activists welcome top court ban on 'sterilization camps' after women's deaths | Reuters. Thomson Reuters Found, 2016. Available: https://www.reuters.com/article/ us-india-women-sterilisation/indian-activists-welcome-top-courtban-on-sterilization-camps-after-womens-deaths-idUSKCN11M1YT [Accessed 30 Sep 2020].

5 IIPS/India II for PS-, ICF. India national family health survey NFHS-4. Published Online First: 1 December 2017, 2015-16. Available: https://dhsprogram.com/publications/publication-FR339-DHS-FinalReports.cfm [Accessed 30 Sep 2020].

6 United Nations. Trends in contraceptive use worldwide. 2015, 2015.

7 Muttreja P, Singh S. Family planning in India: the way forward. Indian J Med Res 2018;148:S1-9.

8 Bruce J. Fundamental elements of the quality of care: a simple framework. Stud Fam Plann 1990;21:61-91.

9 Holt K, Dehlendorf C, Langer A. Defining quality in contraceptive counseling to improve measurement of individuals' experiences and enable service delivery improvement. Contraception 2017;96:133-7.

10 Jain AK, Hardee K. Revising the FP quality of care framework in the context of rights-based family planning. Stud Fam Plann 2018;49:171-9.

11 Tumlinson K. Measuring quality of care: A review of previously used methodologies and indicators. Reprod Health (Published Online First: 1 January 2016).

12 Ravindran TKS. Rural women's experiences with family welfare services in Tamil Nadu. In: Improving Quality of Care in India's Family Welfare Programme. New York: Population Council, 1999: 70-91.

13 Achyut P, Nanda P, Khan N, et al. Quality of care in provision of female sterilization and IUD services: an assessment study in Bihar. New Delhi: International Center for Research on Women, 2014. 
14 Holt K, Zavala I, Quintero X, et al. Women's preferences for contraceptive counseling in Mexico: results from a focus group study. Reprod Health 2018;15:128.

15 Dehlendorf C, Levy K, Kelley A, et al. Women's preferences for contraceptive counseling and decision making. Contraception 2013;88:250-6.

16 Hindin MJ, McGough LJ, Adanu RM. Misperceptions, misinformation and myths about modern contraceptive use in Ghana. J Fam Plann Reprod Health Care 2014;40:30-5.

17 Dehlendorf C, Krajewski C, Borrero S. Contraceptive counseling: best practices to ensure quality communication and enable effective contraceptive use. Clin Obstet Gynecol 2014;57:659-73.

18 Holt K, Zavala I, Quintero X, et al. Development and validation of the Client-Reported quality of contraceptive counseling scale to measure quality and fulfillment of rights in family planning programs. Stud Fam Plann 2019;50:137-58.

19 Ritchie J, Spencer L, Spencer L. Qualitative data analysis for applied policy research. Anal. Qual. Data 2002:187-208.
20 Kruk ME, Gage AD, Arsenault C, et al. High-quality health systems in the sustainable development goals era: time for a revolution. Lancet Glob Health 2018;6:e1196-252.

21 Hardee K, Harris S, Rodriguez M, et al. Achieving the goal of the London summit on family planning by adhering to voluntary, rightsbased family planning: what can we learn from past experiences with coercion? Int Perspect Sex Reprod Health 2014;40:206-14.

22 Senderowicz L. "I was obligated to accept": a qualitative exploration of contraceptive coercion. Soc Sci Med 2019;239:112531.

23 Family Planning. Fam. Plan. 2020. 2020, 2020. Available: https:// www.familyplanning2020.org/ [Accessed 30 Sep 2020].

24 Jungari S, Sharma B, Wagh D. Beyond maternal mortality: a systematic review of evidences on mistreatment and Disrespect during childbirth in health facilities in India. Trauma Violence Abuse 2021;22:739-51.

25 Holt K, Caglia JM, Peca E, et al. A call for collaboration on respectful, person-centered health care in family planning and maternal health. Reprod Health 2017;14:20. 